6. Borst, G.C., Michenfelder, H.J. \& O'Brian, J.T. Discordant cortisol response to exogenous ACTH and insulin-induced hypoglycaemia in patients with pituitary disease. $N$ Engl $J$ Med 1982, 306: 1462-1464.

7. Cunningham, S.K., Moore, A. \& McKenna, J. Normal cortisol response to corticotropin in patients with secondary adrenal failure. Arch Intern Med 1983, 143: 2276-2279.

8. Tsatsoulis, A., Shalet, S.M., Harrison, J. et al. Adrenocorticotrophin (ACTH) deficiency undetected by standard dynamic tests of the hypothalamic-pituitary-adrenal axis. Clin Endocrinol 1988, 28: 225-232.

\section{Percutaneous endoscopic gastrostomy in elderly patients}

Sir,

Finucane et al. ${ }^{1}$ report one death from peritonitis in a series of 28 percutaneous endoscopic gastrostomy tube (PEG) insertions. This was attributed to laceration of the stomach, shown at post mortem examination. The most likely explanation appears to have been trauma, caused while the patient was in a state of agitation, from pulling at the gastrostomy tube. Laceration of the stomach may also occur during the procedure of PEG insertion and may result in the development of symptoms and signs of peritoneal irritation. We discuss one such case below. The technique we use for PEG insertion is similar to that described by Finucane et al. ${ }^{1}$

A 27 year old woman with cystic fibrosis was referred for PEG insertion because of deteriorating nutritional state despite intermittent feeding via a fine-bore nasogastric tube. In view of poor lung function, minimum sedation was used for the procedure. Initially, she was given intravenous midazolam $3 \mathrm{mg}$ and cefuroxime $750 \mathrm{mg}$, and the skin, abdominal wall and stomach infiltrated with $2 \%$ lignocaine solution. During endoscopic intubation, the patient began retching and struggled. She remained agitated despite being given additional sedation. The percutaneous cannula (Fresenius AG, Bad Homburg, Germany) was introduced without difficulty, with a single pass. Immediately after the procedure the patient complained of severe abdominal pain and was given omnopon $5 \mathrm{mg}$ intramuscularly. Six hours later, when water was introduced via the gastrostomy, the patient complained of abdominal pain. The pain persisted for a few hours and was exacerbated by movement. Examination revealed generalized guarding with rebound tenderness in the epigastrium. Bowel sounds were present but diminished. A plain abdominal radiograph revealed free gas under the left hemi-diaphragm but no radio- $\frac{2}{2}$ graphic evidence of ileus. She was given intravenous fluids $\mathbb{D}$ and antibiotics and nothing by mouth. Over the next $24 \stackrel{2}{c}$ hours the pain improved and signs of guarding and

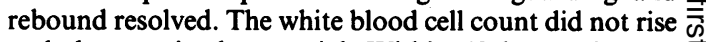
and she remained apyrexial. Within 48 hours she was taking oral fluids. Feeding through the gastrostomy tube was commenced 3 days later.

The case we describe is consistent with the development of peritoneal irritation as a consequence of laceration of $\mathbb{Q}$ the stomach, during the procedure of PEG insertion. In view of this, whenever patients experience discomfort on first passage of fluid through the PEG tube, we have adopted a policy of deferring its use by 12-24 hours. In $\overrightarrow{\vec{H}}$ considering ways of reducing a low complication rate $\vec{O}_{\vec{O}}$ from PEG further, ${ }^{2-4}$ we emphasize the importance of $\frac{}{0}$ adequate sedation to cover PEG insertion. Well-sedated patients are less likely to struggle during the procedure and, as a consequence, the risk of laceration of the $\infty$ stomach should be diminished. Improved monitoring 0 during endoscopic procedures with pulse-oxymetry, and $O$ general availability of opiate and benzodiazepine $\varphi$ antagonists may diminish the risks from oversedation.

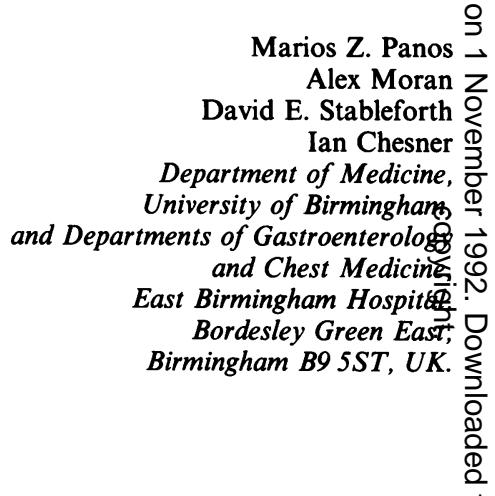

1. Finucane, P., Aslan, S.M. \& Duncan, D. Percutaneous $\overrightarrow{\vec{O}}$ endoscopic gastrostomy in elderly patients. Postgrad Med J 1991, 67: 371-373.

2. Mamel, J.J. Percutaneous endoscopic gastrostomy. Am J Gastroenterol 1989, 84: 703-710.

3. Miller, R.E., Castlemain, B., Lacqua, F.J. \& Kotler, D.P. Percutaneous endoscopic gastrostomy. Results in 316 patients 3 and review of the literature. Surg Endosc 1989, 3: 186-190.

4. Larson, D.E., Barton, D.D., Schroeder, K.W. \& Dimagno, E.P. Percutaneous endoscopic gastrostomy: indication, success, complications and mortality in 314 consecutive patients. Gastroenterology 1987, 93: 48-52. 